\title{
fMRI on Affective Stimuli in Patients with Paranoid Schizophrenia
}

\author{
Arkhipov AY ${ }^{1 *}$, Maslennikova $\mathrm{AV}^{1}$, Ushakov $\mathrm{VL}^{2}$ and Strelets $\mathrm{VB}^{1}$ \\ ${ }^{1}$ Institute of Higher Nervous Activity and Neurophysiology of RAS, Russia \\ ${ }^{2}$ National Research Center Kurchatov Institute, Moscow, Russia
}

Submission: August 10, 2017; Published: August 24, 2017

*Corresponding author: Arkhipov AY, Institute of Higher Nervous Activity and Neurophysiology of RAS, Russia, Email: arhip76@bk.ru/vbstrelets@gmail.com

\section{Abstract}

We studied fMRI to neutral and negative emotional stimuli in schizophrenics with hallucinatory-paranoid syndrome in implicit situation to find the brain structures activated by these two categories of stimuli. It was revealed that in patients the number of activated structures was greater than in norm especially to neutral stimuli in limbic structures. The obtained results point to excessive and not precise reaction of the brain structures possibly caused by the decrease of central inhibition processes in schizophrenia.

Keywords: Schizophrenia; Hallucinatory-paranoid syndrome; Neuroimaging, fMRI

Abbreviations: ERP: Event Related Potentials; fMRI -Functional Magnetic Resonance Imaging; PANSS: Positive and Negative Syndrome Scale; IAPS: International Affective Picture System; ICD-10- International Classification of Diseases $10^{\text {th }}$

\section{Introduction}

The purpose of our study was to determine what brain structures are activated by stimuli of different emotional significance in implicit situation in patients in comparison with normal control obtained under the same conditions (fMRI) [13]. Our previous ERP [4] analysis showed that patients with pseudo-hallucinations, as a symptom of affective perception pathology can be linked to malfunction of physiological brain reactions to the same emotional negative stimuli as used in fMRI study. The comparison of the results of the brain structures activation, obtained by neuroimaging and ERP methods can help to understand the mechanisms of hallucinatory-paranoid syndrome $[5,6]$.

\section{Participants and Methods}

Patients with schizophrenia F 20.0 in the ICD-10 without neuroleptic therapy and cerebral trauma, aged 20 to 40 years ( $n=6,2$ women, 4 men). The value of positive symptoms for PANSS was $97.1 \pm 3.1$. As a control group, subjects $(n=5$, 2 women, 3 men) without psychiatric disorders, aged 18 to 35 years were examined. All participants were right-handed, without neurological disorders. Stimuli: visual stimuli were pictures from IAPS base, adopted for Russian sample, different in emotional meaning-"threatening" and "neutral" (total 80 pictures, 40 in each category). There were landscapes, flowers and other positive things as neutral pictures and explosions, weapons, damages as threatening stimuli. Tomography and fMRI data was obtained on 3T tomography Magnetom Verio, Siemens. The regions are defined according to the MNI atlas. ERPs were recorded to the same participants and stimuli according to standard protocol.

(hallucinatory-paranoid syndrome) with the first episode,

Table 1: Main regions of fMRI activation at fMRI on stimuli of different affective significance.

\begin{tabular}{|c|c|c|}
\hline & SCH & Norm \\
\hline «Threatening» category & $\begin{array}{c}\text { Prefrontal cortex (left) Cingulate gyrus } \\
\text { (anterior) }\end{array}$ & \\
\hline \multirow{3}{*}{ «Neutral» category } & $\begin{array}{c}\text { Temporal cortex (left) Precuneus (right) } \\
\text { Thalamus Hippocampus Hipothalamus } \\
\text { Amigdala }\end{array}$ & $\begin{array}{c}\text { Frontal cortex (left) Orbital cortex } \\
\text { Temporoparietal cortex (left) }\end{array}$ \\
\hline
\end{tabular}


Results

The results are shown in Figure 1 and in the Table 1. With neutral stimuli more activated subcortical structures appeared in the group of patients while in norm more activated were temporo-parietal-occipital and frontal areas of the left hemisphere and orbital cortex. More activated areas in response to threatening stimuli in norm became prefrontal area of the left hemisphere and anterior part of gyrus cingulate while in patients it was only temporo-parietal-occipital area of the left hemisphere.

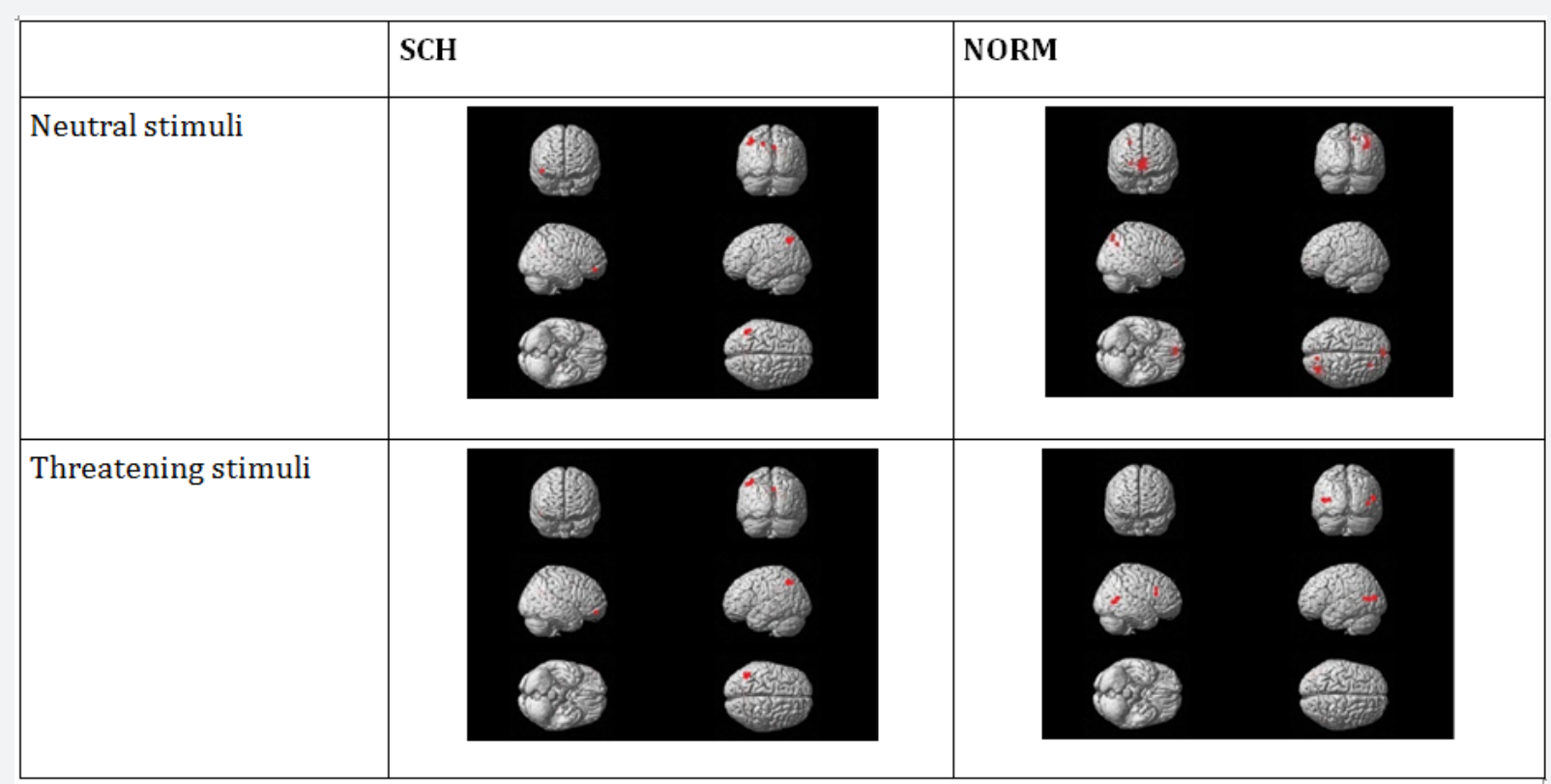

Figure 1: Active regions on two groups of stimuli in patients and control.

In ERP study in patients with paranoid schizophrenia we found the increase of both parameters - latency and amplitude of P200, P300 and N400 components to negative emotional stimuli in comparison with neutral ones and called this phenomenon paradox effect.

\section{Discussion}

Thus, our fMRI study shows that the limbic structures to neutral stimuli in schizophrenic patients are hyperactivated while the cortical ones - hypoactivated, pointing to decreased level of cortical inhibition in prefrontal and temporoparietal areas of the left hemisphere in paranoid schizophrenia. The brain structures reaction to biologically more important (threatening) stimuli in norm includes only the activation of temporo-parietaloccipital areas due to the preliminary brain analysis of the neutral (background) stimuli. However, malfunction of the background stimuli analysis in patients evidently requires the additional activation of prefrontal areas of the left hemisphere and cingulate gyrus during perception of the threatening stimuli. This additional activation obtained in patients by fMRI method coincides with their physiological results-simultaneous increase of both of ERPs components parameters, amplitude and latency, to the threatening stimuli in comparison with the neutral ones that we called paradox effect. We suppose that these additional components revealed by fMRI and ERPs methods may link to "additional" hallucinatory-paranoid syndrome.

\section{Conclusion}

i. Our neuroimaging study shows hyperactivation of limbic and hypoactivation of the highest cortical structures to emotionally neutral and the opposite results to biologically significant (emotional negative) stimuli in patients with paranoid schizophrenia.

ii. Our ERPs study reveals in patients paradox effect simultaneous increase of both ERP parameters - latencies and amplitudes to biologically significant stimuli.

iii. We suppose that additional effects revealed in patients with paranoid schizophrenia by fMRI and ERP methods can link to positive "additional" symptoms revealed in these patients by PANSS.

\section{Acknowledgement}

Thanks for the help in preparing this review researches of NRC "Kurchatov Institute" Kartashev Sergey and Orlov Vacheslav.

\section{Conflict of Interest}

This work was supported by the RFBR grant No. 15-0604303.

\section{References}

1. Takahashi H, Koeda M, Odaetal K (2004) An fMRI study of differential neural response to affective pictures in schizophrenia. Neurolmage 22(3): 1247-1254. 
2. Gur RE, McGrath C, Chan RM, Schroeder L, Turner T, et al. (2002) An fMRI study of facial emotion processing in patients with schizophrenia. American Journal of Psychiatry 159(12): 1992-1999.

3. Reske M, Habel U, Kellermann T, Backes V, Jon Shah N, et al. (2009) Differential brain activation during facial emotion discrimination in first-episode schizophrenia. J Psychiatr Res 43(6): 592-599.

4. Strelets VB, Arkhipov AY (2015) The Influence of Threatening Stimuli on the Component P200 in Patients with Paranoid Schizophrenia Fiziol Cheloveka 41(5): 66-73.
5. Taylor SF, Liberzon I, Decker LR, Koeppe RA (2002) A functional anatomic study of emotion in schizophrenia. Schizophrenia Research 58(2-3) 159-172.

6. Nadia L, Adrianna M (2013) Individuals Diagnosed with Schizophrenia Assign EmotionalImportance to Neutral Stimuli: An fMRI Study. ISRN Psychiatry.
Your next submission with Juniper Publishers will reach you the below assets

- Quality Editorial service

- Swift Peer Review

- Reprints availability

- E-prints Service

- Manuscript Podcast for convenient understanding

- Global attainment for your research

- Manuscript accessibility in different formats

( Pdf, E-pub, Full Text, Audio)

- Unceasing customer service

Track the below URL for one-step submission https://juniperpublishers.com/online-submission.php 